\title{
Commutative Banash algebras with non-unique complete norm topology
}

\section{Richard J. Loy}

\begin{abstract}
It has recently been shown that discontinuous functional calculi exist for certain commutative Banach algebras. Such an algebra thus possesses two distinct calculi so that there exist analytic functions whose action on the algebra is not uniquely determined. In this note a method is given for constructing commutative Banach algebras which admit two inequivalent complete norm topologies and the result is applied to show that the action of any non-algebraic analytic function may fail to be uniquely defined.
\end{abstract}

The purpose of this paper is to give a simple method for constructing commutative Banach algebras which have non-unique complete norm topology. One of the first examples of such an algebra was that of Feldman (see [1], an example originally constructed to show the failure of the Wedderburn Theorem. This algebra is $l_{2} \oplus C$ as a vector space with the usual product in $Z_{2}$ and trivial multiplication by the second summand, and has a norm in which $\tau_{2}$ is dense. Thus in a sense $\tau_{2}$ has been completed by the adjunction of a radical and the resulting algebra has the non-uniqueness property. This is the idea exploited herein, and although the norm of the Feldman example is not obtainable by our approach other inequivalent norms on $z_{2} \oplus \mathrm{C}$ may be constructed. Indeed this is essentially done in [1].

\section{1.}

Let $A$ be a commutative normed algebra and $M$ a commutative Banach Received 11 February 1974. 
$A$-module, hereafter simply $A$-module, so that $M$ is a Banach space which is a commutative $A$-module such that the map $(x, m) \mapsto x \cdot m$ is continuous from $A \times M$ to $M$. For such $A$ and $M$ let $H^{l}(A, M)$ denote the first algebraic cohomology group, $H_{c}^{l}(A, M)$ the first continuous cohomology group, where the cochains are required to be bounded. Thus with the usual conventions $H^{I}(A, M)$ is the space of derivations of $A$ into $M$, that is, linear mappings $D: A \rightarrow M$ satisfying $D(x y)=x \cdot D y+y \cdot D x ; H_{c}^{I}(A, M)$ the space of continuous derivations of $A$ into $M$. As a simple example take $M=C$ with module action $x \cdot \lambda=\lambda \varphi(x)$ for some multiplicative linear functional $\varphi$ on $A$. If $\varphi \neq 0$ then $H^{l}(A, C)$ is the point derivation space at $\varphi$, if $\varphi=0$ then it is the annihilator of $A^{2}$. This situation is considered in [1]. For further details of Banach algebra cohomology see [6] or [7].

Denoting the norms in both $A$ and $M$ by $\|\cdot\|$, the constarit

$$
k=\sup \{1,\|x \cdot m\|:\|x\|=\|m\|=1\}
$$

is finite, and if $\bar{A}$ is the completion of $A$ under $\|\cdot\|, M$ is clearly an $\bar{A}$-module. Let $A$ denote the vector space direct sum $\bar{A} \oplus M$ with product

$$
(x, m)(y, n)=(x y, x \cdot n+y \cdot m)
$$

and norm

$$
|(x, m)|=k(\|x\|+\|m\|)
$$

For each $D \in H^{1}(A, M)$ the functional

$$
|\cdot|_{D}:(x, m) \mapsto k(\|x\|+\|D x-m\|)
$$

is defined on the subalgebra $A \oplus M$ of $A$ and is easily seen to be an algebra norm thereon. If $D=0$ the completion of $A \oplus M$ under $|\cdot|_{D}$ is of course just A. For general $D$ we note that the map

$$
\theta_{D}:(x, m) \mapsto(x, D x-m)
$$

is an isometric isomorphism of $A \oplus M$ under $|\cdot|_{D}$ into $A$, and so 
extends uniquely to a map of the completion $A_{D}$ of $A \oplus M$ into $A$. In particular if $1: x \mapsto(x, 0)$ is the natural embedding of $A$ into $A \oplus M$ then $\|\cdot\|_{D}: x \mapsto|l(x)|_{D}$ is a norm on $A$ and $\theta_{D}$ extends to an isometric isomorphism of $\bar{A}_{D}$, the completion of $A$ under $\|\cdot\|_{D}$, with $\overline{\operatorname{Gr}(D)}$, the closure (in $A$ ) of the graph of $D$.

Now if $D$ is continuous then $|\cdot|_{D}$ is equivalent to $|\cdot|$ on $A \oplus M$ and $\|\cdot\|_{D}$ is equivalent to $\|\cdot\|$ on $A$. Thus $A_{D}=A$ and $\bar{A}_{D}=\bar{A}$. In the discontinuous case $\|\cdot\|_{D}$ is a discontinuous norm on $A$ and $l$ is a discontinuous isomorphism. This latter result has been used in [1], in the case $A=Z_{p}$ for $1 \leq p<\infty$ and $M=C$ with trivial action, to show that these algebras admit discontinuous isomorphisms. Another example is furnished by letting $A$ be the algebra of polynomials on the unit disc with uniform norm, $M=C$ with module action $p \cdot \lambda=\lambda p(1)$ for $(p, \lambda) \in A \oplus M$ and $D: p \mapsto p^{\prime}(1)$. Here $\bar{A}_{D}$ is a one generator algebra with spectrum the closed unit disc and one dimensional radical (cf. [5]).

Suppose now that $D$ is discontinuous. If $M$ is finite dimensional then it easily follows that $\overline{\operatorname{Gr}(D)} \cap 0 \oplus M \neq\{0\}$, and if $A$ is complete this holds for arbitrary $M$ by the closed graph theorem. Thus if $A$ is a Banach algebra with $H^{1}(A, M) \neq H_{C}^{l}(A, M)$ for some $M$ then $A$ has a completion with a non-trivial nil ideal. We remark that this fails for $A=C(X)$ since $H^{1}(C(X), M)=0$ for any $C(X)$-module $M$ ([6], [7]). Other cases in which $H^{I}(A, M)=H_{c}^{1}(A, M)$ are given in [2]. Finally we add one further hypothesis to these considerations to obtain the main result.

THEOREM 1. Let $A$ be a commutative Banach algebra, $D$ a non-zero derivation of $A$ into a conmutative Banach A-module $M$. If $D$ vanishes on a dense subset of $A$ then the algebra $\bar{A}_{D}$ admits two inequivalent complete norm topologies.

Proof. Let $S=\{m \in M:(0, m) \in \overline{\mathrm{Gr}(D)}\}$ so that $S$ is certainly contained in $\overline{D(A)}$. Conversely, if $m=D x$ for some $x \in A$ take $\left\{x_{n}\right\} \subset D^{-1}(0)$ with $x_{n}+x$ in $A$. Then $x-x_{n} \rightarrow 0, D\left(x-x_{n}\right)=m$ 
whence $m \in S$. A diagonal argument shows that $S$ is closed and so $S=\overline{D(A)}$. But then if $(x, m) \in A \oplus S$ it follows that $m-D x \in \overline{D(A)}$ so that $(0, m-D x) \in \overline{\operatorname{Gr}(D)}$ and $(x, m)=(x, D x)+(0, m-D x) \in \overline{\operatorname{Gr}(D)}$. Similarly $\operatorname{Gr}(D) \subset A \oplus S$ and since the latter is closed in $A$ we have $A \oplus S=\overline{\operatorname{Gr}(D)}$.

Now if $(x, m) \in A \oplus S$ then $\theta_{D}(x, m)=(x, 0)+(0, D x-m) \in A \oplus S$. Conversely $(x, m)=(x, D x-m)+(0,2 m-D x) \in \theta_{D}(A \oplus S)$. Thus $\theta_{D}$ is an automorphism of $A \oplus S$. Since $D$ is discontinuous so is $\theta_{D}$ and so the norms $|\cdot|$ and $|\cdot|_{D}=\left|\theta_{D}(\cdot)\right|$ are inequivalent on $A \oplus S$. The completeness of $|\cdot|$ is clear and that of $|\cdot|_{D}$ follows since $\theta_{D}$ is an automorphism. Since $\left(\bar{A}_{D},\|\cdot\|_{D}\right)$ is (isometrically) isomorphic to $(A \oplus S,|\cdot|)$ the result follows.

As an application of this method let $A$ be the algebra of functions continuous on the closed unit disc $\Delta$ and analytic on its interior, with the uniform norm. Let 0 be the algebra of functions analytic on a neighbourhood of $\Delta$, and $\psi: A \rightarrow 0$ the monomorphism $\psi x(\lambda)=x(\lambda / 2)$, $x \in A,|\lambda|<2$. Finally let $M$ be a Banach space, $T$ an endomorphism of $M$ with norm at most one. For $x \in A, x(\lambda)=\sum \mu_{n} \lambda^{n},|\lambda|<1$, the series $\sum \mu_{n} 2^{-n} T^{n}$ converges to $\psi x(T)$, the operator on $M$ given by the functional calculus applied to the function $\psi x$ and the operator $T$. It follows that

$$
m \cdot x=x \cdot m=\sum_{n} \mu_{n} 2^{-n} T^{n} m
$$

defines a commutative module action of $A$ on $M$. Further

$$
\begin{aligned}
\|x \cdot m\| & \leq \sum\left|\mu_{n}\right| 2^{-n}\|m\| \\
& =\sum\left|\mu_{n}\right| 2^{-n / 2} \cdot 2^{-n / 2}\|m\| \\
& \leq\left(\sum\left|\mu_{n}\right|^{2} \cdot 2^{-n}\right)^{1 / 2} \sqrt{2}\|m\| \\
& \leq \sqrt{ } 2\|x\| \cdot\|m\|
\end{aligned}
$$

so that $M$ is an $A$-module. 
In particular, we may consider the situation developed in [3], where $M=C[0,1]$ and $T$ is the operator of indefinite integration. Non-zero linear mappings $B: O \rightarrow M$ are constructed in [3] to vanish on polynomials and satisfy

$$
B(f g)=f(T) B(g)+g(T) B(f)
$$

for $f, g \in 0$. Letting $D=\beta \psi$ we have $D: A \rightarrow M$ is a derivation vanishing on polynomials. Since $D \neq 0$ it is necessarily discontinuous, which answers a question of [2].

We also note that $A$ and $D$ here satisfy the hypothesis of Theorem 1 since polynomials are dense in $A$. Thus $\bar{A}_{D}$ has two inequivalent complete norm topologies. In [3] the question is raised as to whether the exponential function in such an algebra is independent of the norm and using the present example we settle this in the negative. Indeed, let $f \in 0$ be non-algebraic. In the terminology of [3], $\tilde{p}$ consists of the rational functions in $O$ so that $\psi f$ is not algebraic over $\tilde{P}$. Thus the mapping $B$ may be chosen such that $D f=\beta \psi(f) \neq 0$. Let $p_{n}$ be the $n$-th partial sum of the Taylor expansion of $f$ about zero, so that $p_{n} \rightarrow f$ uniformly on some neighbourhood of $\Delta$, and denote by $j$ the identity function on $\Delta$ so that the spectrum of $(j, 0)$ in $A \oplus S$ is $\Delta$. Let $f(j)$ and $f_{D}(j)$ respectively denote the elements of $A \oplus S$ obtained from $(j, 0)$ by $f$ under the $|\cdot|$-continuous and $|\cdot|_{D}$-continuous functional calculi. Then $p_{n}((j, 0))=\left(p_{n}, 0\right) \rightarrow f(j)$ under $|\cdot|,\left(p_{n}, 0\right) \rightarrow f_{D}(j)$ under $|\cdot|_{D}$. Since $\left|\left(p_{n}, 0\right)-(f, 0)\right| \rightarrow 0$ it follows that $f(j)=(f, 0)$, but $\left|\left(p_{n}, 0\right)-(f, 0)\right|_{D}=\left\|p_{n}-f\right\|+\|D f\|+0$ so that $f_{D}(j) \neq f(j)$.

We remark that by the construction of $B$ (see [3]) the radical of $\bar{A}_{D}$ is infinite dimensional. Indeed algebras with finite dimensional radical have unique functional calculus by Theorem 1 of [3] so that the argument above shows that any derivation of $A$ into a finite dimensional $A$-module which vanishes on polynomials must be zero. In fact more is true: any derivation of $A$ into a finite dimensional $A$-module is continuous. For if $D: A+M$ is such a derivation then $A \oplus M$ is a strongly decomposable Banach algebra with finite dimensional radical $M, A$ is an algebra of 
class $B$ (see [4]) and so the isomorphism $\theta_{D^{l}}$ is continuous by Theorem 5.4 of [4], and so $D$ is continuous.

2.

In the above situations the ideal adjoined was always nilpotent of index two; we now consider how to obtain more general ideals. Let $A$ be a commutative normed algebra, $M$ an $A$-module which is also a commutative Banach algebra, $D=\left\{D_{1}, \ldots, D_{r}\right\}$ a higher derivation of rank $r$ of $A$ into $M$, so that for $x, y \in A$ and $1 \leq s \leq r$,

$$
D_{s}(x y)=x \cdot D_{s} y+y \cdot D_{s} x+\sum_{i=1}^{s-1} D_{i}(x) D_{s-i}(y) \text {. }
$$

In analogy to Section 1 let $A_{r}$ denote the vector space $\bar{A} \oplus M^{r}$ with product

$$
\left(x,\left\{m_{i}\right\}\right)\left(y,\left\{n_{i}\right\}\right)=\left(x y,\left\{x \cdot n_{i}+y \cdot m_{i}+\sum_{j=1}^{i-1} m_{j} n_{i-j}\right\}\right)
$$

and norm

$$
\left|\left(x,\left\{m_{i}\right\}\right)\right|=k\|x\|+k \sum_{i=1}^{r}\left\|m_{i}\right\|
$$

We also have the norm $|\cdot|_{D}$ on $A \oplus M^{r}$,

$$
\left|\left(x,\left\{m_{i}\right\}\right)\right|_{D}=k\|x\|+k \sum_{i=1}^{r}\left\|D_{i} x-m_{i}\right\|,
$$

the isomorphism $\theta_{D}:\left(x,\left\{m_{i}\right\}\right) \rightarrow\left(x,\left\{D_{i} x-m_{i}\right\}\right)$ and the completion $\bar{A}_{D}$ of $A$ under \|\|$_{D}: x \mapsto|l(x)|_{D}$. A detailed analysis of $\bar{A}_{D}$ depends on the properties of $D=\left\{D_{1}, \ldots, D_{p}\right\}$ and for this reason we consider $a$ specific case only. Note, however, that if $D_{1}, \ldots, D_{r-1}$ are continuous but $D_{p}$ is discontinuous then the arguments of Section 1 are applicable.

Thus let $A$ be the algebra of polynomials on the closed unit disc $\Delta$, with uniform norm $\|\cdot\|, M=C$ with module action as before and higher 
derivation $D=\left\{D_{1}, \ldots, D_{r}\right\}$ given by

$$
D_{i} p=\frac{p^{(i)}(1)}{i !}
$$

so that

$$
\|p\|_{D}=\|p\|+\sum_{i=1}^{r} \frac{\left|p^{(i)}(1)\right|}{i !} .
$$

For positive integers $s, t$ set

$$
p_{s t}(\lambda)=\frac{(1+\lambda)^{s}}{2^{s}} \cdot \frac{(1-\lambda)^{t}}{t !}
$$

so that for $|\lambda| \leq 1$ and $0<\delta<1$,

$$
\left|p_{s t}(\lambda)\right| \leq \begin{cases}\delta & |1-\lambda| \leq \delta, \\ \frac{2^{t}}{t}\left(\frac{4-\delta^{2}}{4}\right)^{s / 2} & |1-\lambda|>\delta,\end{cases}
$$

whence $\left\|p_{s t}\right\| \rightarrow 0$ as $s \rightarrow \infty$ for each integer $t$. Also

$$
p_{s t}^{(i)}(1)= \begin{cases}0 & i<t, \\ 1 & i=t .\end{cases}
$$

We wish to show that given $\alpha_{1}, \ldots, \alpha_{r} \in C$ there is a sequence $\left\{p_{n}\right\} \subset A$ with $\left\|p_{n}\right\| \rightarrow 0$ and $D_{i} p_{n}+\alpha_{i}$ for each $i$. Rather than giving an involved construction of such a sequence we proceed as follows. Suppose the subspace $\quad H=\cap D_{i}^{-1}(0)$ of $A$ is not dense, so there is a continuous functional $\varphi$ on $A$ vanishing on $H$ but not identically zero. But such $\varphi$ must be of the form $\sum \lambda_{i} D_{i}$ for some $\lambda_{1}, \ldots, \lambda_{r} \in C$. Since $\varphi \neq 0$ some $\lambda_{i} \neq 0$, but then if $\lambda_{t}$ is the last such scalar $\lambda_{t}=\varphi\left(p_{s t}\right) \rightarrow 0$ as $s+\infty$. This contradiction shows that $B$ is dense in $A$. Since $D_{1}, \ldots, D_{r}$ are linearly independent there exist $q_{i}, \ldots, q_{r} \in A$ with $D_{i} q_{j}=\delta_{i j}$ whence the set $\left\{p: D_{i} p=\alpha_{i}, i=1, \ldots, r\right\}$ is a translate of $B$ and so is dense in $A$. The existence of the required sequence 
$\left\{p_{n}\right\}$ is now clear. Now let $\theta$ denote the extension by continuity of the isomorphism $\theta_{D^{l}}$ to $\bar{A}_{\mathrm{D}}$. Then if $\left(x,\left\{\alpha_{i}\right\}\right) \in A \oplus M^{r}$ we have $\left(x,\left\{\alpha_{i}\right\}\right)=\left(x,\left\{D_{i} x\right\}\right)+\left(0,\left\{\alpha_{i}-D_{i} x\right\}\right) \in \theta\left[\bar{A}_{D}\right)$, and since $\theta$ is an isometry and $\bar{A}_{D}$ is complete it follows that $\theta$ maps $\bar{A}_{D}$ onto $A_{r}=\bar{A} \oplus M^{r}$. Thus $\bar{A}_{D}$ has a radical which is nilpotent of index $r$.

We note that in this example $A_{r}$, and hence $\bar{A}_{D}$, is a strongly decomposable Banach algebra of class $B$ with finite dimensional radical and so has unique complete norm topology by Theorem 5.4 of [4]. In comparison to this we note the following result the proof of which is omitted.

THEOREM 2. Let $A$ be a commutative Banach algebra, $D=\left\{D_{1}, \ldots, D_{r}\right\}$ a higher derivation of rank $r$ of $A$ into $C$ such that $\left\{D_{1}, \ldots, D_{p}\right\}$ is a linearly independent set of discontinuous functionals. Then $\bar{A}_{\mathrm{D}}$ admits two inequivalent complete norm topologies and has nilpotent elements of index $r$.

These results naturally raise the question of whether quasinilpotent non-nilpotent radicals are obtainable in some analogous fashion. As yet we have been unable to settle this question; however some partial results follow for the particular case when $A$ is the algebra of polynomials on the closed unit disc $\Delta$. Let $\left\{M_{k}\right\}$ be a sequence of positive reals such that

$$
\frac{M_{k}}{k !} \geq \frac{M_{i}}{i !} \cdot \frac{M_{k-i}}{(k-i) !}
$$

for $1 \leq i<k$ so that, in particular, the sequence $k \mapsto\left(\frac{k !}{M_{k}}\right)^{1 / k}$ is monotonic decreasing, with limit $\gamma \geq 0$. Consider the norm on $A$ given by

$$
|p|=\|p\|+\sum_{1}^{\infty} \frac{\left|p^{(k)}(1)\right|}{M_{k}}
$$

and let $\bar{A}_{\infty}$ be the completion of $A$ under $|\cdot|$. 
THEOREM 3. $\bar{A}_{\infty}$ is semisimple if $\gamma>0 .(A,|\cdot|)$ is natural if and only if $\gamma=0$.

Proof. Suppose $\gamma>0$ and let

$$
\Delta_{\gamma}=\Delta U\{\lambda:|1-\lambda| \leq \gamma\} \text {. }
$$

Then

$$
\begin{aligned}
|p| & =\|p\|+\sum_{1}^{\infty} \frac{\left\lfloor p^{(k)}(1)\right\rfloor}{M_{k}} \\
& \geq\|p\|+\sum_{1}^{\infty} \frac{\left.\mid p^{(k)}(1)\right\rfloor}{k !} \gamma^{k} \\
& \geq \sum_{0}^{\infty} \frac{\mid p(k)(1)\rfloor}{k !} \gamma^{k},
\end{aligned}
$$

so that

$$
|p| \geq \max \left\{|p(\lambda)|: \lambda \in \Delta_{\gamma}\right\}
$$

Thus $(A,|\cdot|)$ fails to be natural since evaluation at any point of $\Delta_{\gamma} \backslash$ is $|\cdot|$-continuous.

Suppose now that $x \in \bar{A}_{\infty}, \nu(x)=0$, and let $\left\{p_{n}\right\} \subset A$ be a sequence converging to $x$. Then certainly $\nu\left(p_{n}\right) \rightarrow 0$ so that $p_{n} \rightarrow 0$ uniformly on $\Delta_{\gamma}$. Thus $p_{n}^{(k)}(1) \rightarrow 0$ as $n \rightarrow \infty$ for each $k \geq 0$. Also the sequence $\left\{p_{n}^{(k)}(1)\right\}_{k \geq 1}$ is Cauchy in $l^{1}\left(\left\{M_{k}^{-1}\right\}\right)$ whence

$$
\lim \sum_{k} \frac{\left|p_{n}^{(k)}(1)\right|}{M_{k}}=\sum_{k} \lim _{n} \frac{\left|p_{n}^{(k)}(1)\right|}{M_{k}}=0 \text {. }
$$

But then $|x|=\underset{n}{\lim }\left|p_{n}\right|=0$ and it follows that $\bar{A}_{\infty}$ is semisimple.

$$
\text { Conversely, if } \gamma=0 \text { let } \alpha \in C,|\alpha|>I \text { and take } r>0 \text { so }
$$
small that $\alpha \vDash \Delta_{r}$. Then the sequence $k \mapsto \frac{k ! 2^{k}}{M_{k} r^{k}}$ is bounded, by $B_{r}$ say, so that 


$$
\begin{aligned}
|p| & \leq\|p\|+B_{r} \sum_{1}^{\infty} \frac{\left|p^{(k)}(1)\right|}{k !}\left(\frac{r}{2}\right)^{k} \\
& \leq\|p\|+B_{r}\left\{\sum_{1}^{\infty}\left[\frac{\left|p^{(k)}(1)\right|}{k !}\left(\frac{r}{\sqrt{2}}\right)^{k}\right)^{2}\right\}^{1 / 2} \\
& \leq C_{r} \max \left\{|p(\lambda)|: \lambda \in \Delta_{r}\right\}
\end{aligned}
$$

for some constant $C_{r}>0$. But then there are polynomials $\left\{p_{n}\right\} \subset A$ with $\max \left\{\left|p_{n}(\lambda)\right|: \lambda \in \Delta_{r}\right\} \leq 1$ for all $n$ and $\left|p_{n}(\alpha)\right| \rightarrow \infty$. Thus evaluation at $\alpha$ is not $|\cdot|$-continuous.

We remark in passing on the similarity between the above expression for $|\cdot|$ and the inequalities for quasianalytic functions on $\Delta$. For taking the norm

$$
|p|^{\prime}=\|p\|+\sum_{1}^{\infty} \frac{\left\|p^{(k)}\right\|}{M_{k}}
$$

on $A$, the completion is the algebra

$$
D\left\{M_{k}\right\}=\left\{x \in C^{\infty}(\Delta): \sum \frac{\left\|x^{(k)}\right\|}{M_{k}}<\infty\right\}
$$

(see [5]). If $\gamma>0$ then the classical class $c\left\{M_{k}\right\}$ is quasianalytic by [9] 4.2.II and hence so is $D\left\{M_{k}\right\}$ (see [8]). In particular if $x \in D\left\{M_{k}\right\}$ and $x^{(k)}(1)=0$ for all $k$ then $x=0$, a result which is more directly seen by appeal to the argument of Theorem 3 . There is no converse, however, since if $M_{k}=k !(\log k)^{k}$ then $D\left\{M_{k}\right\}$ is quasianalytic whereas $\gamma=0$.

We conclude with a simple characterization of the radical in certain completions of the algebra $A$. Let $|\cdot|^{\prime}$ be a norm on $A$ such that $\left(A,|\cdot|^{\prime}\right)$ is natural and denote by $\bar{A}^{\prime}$ the $|\cdot|^{\prime}$-completion of $A$. For $B \subset \bar{A}^{\prime}$ write $\bar{B}^{\prime}$ for its closure. Finally note that the Gel'fand transform in $\bar{A}^{\prime}$ is the identity map on $A$.

THEOREM 4. The radical of $\bar{A}$ ' is precisely $\prod_{k} \bar{j}^{k_{A}^{\prime}}$. 
Proof. Suppose $x \in \overline{n j}^{k_{A}}$ so that for fixed $k$ there is $\left\{p_{n}\right\} \subset A$ with $j^{k} p_{n} \rightarrow x$ in $\bar{A}^{\prime}$. But then $j^{k} p_{n} \rightarrow \hat{x}$ uniformly on $\Delta$. Thus the analytic function $\hat{x}$ has a $k$-th order zero at the origin. Since this is true for all $k$ it follows that $x$ vanishes on $\Delta$, so that $x$ lies in the radical.

Conversely, if $x$ lies in the radical take $\left\{p_{n}\right\} \subset A$ with $p_{n} \rightarrow x$ in $\bar{A}^{\prime}$. Then certainly $p_{n}(0) \rightarrow 0$ so that $p_{n}-p_{n}(0) j \rightarrow x$ in $\bar{A}^{\prime}$, that is, $x \in \overline{j A}$, Now suppose that $x \in \bar{j}^{\prime}{ }^{\prime}$ for some $k \geq 1$. If $\left\{q_{n}\right\} \subset A$ are such that $j^{k} q_{n} \rightarrow x$ in $\bar{A}^{\prime}$ then $j^{k} q_{n} \rightarrow 0$ uniformiy on $\Delta$ whence $q_{n}(0) \rightarrow 0$. But then $x \in \overline{j^{k+1} A}$ as above and the result follows by induction on $k$.

\section{References}

[1] W.G. Bade and P.C. Curtis, Jr., "Homomorphisms of commutative Banach algebras", Amer. J. Math. 82 (1960), 589-608.

[2] W.G. Bade and P.C. Curtis, Jr., "The continuity of derivations of Banach algebras", preprint.

[3] H.G. Dales, "The uniqueness of the functional calculus", Proc. London Math. Soc. (3) 27 (1973), 638-648.

[4] H.G. Dales and J.P. MCClure, "Continuity of homomorphisms into certain commutative Banach algebras", Proc. London Math. Soc. (3) 26 (1973), 69-81.

[5] H.G. Dales and J.P. MCClure, "Completion of normed algebras of polynomials.", preprint.

[6] B.E. Johnson, Cohomology in Banach algebras (Memoirs Amer. Math. Soc., 127. Amer. Math. Soc., Providence, Rhode Island, 1972).

[7] Herbert Kamowitz, "Cohomology groups of commutative Banach algebras", Trans. Amer. Math. Soc. 102 (1961), 352-372. 
[8] B.1. Korenbljum, "Quasianalytic classes of functions in a circle", Soviet Math. DokZ. 6 (1965), 1155-1158.

[9] S. Mandelbrojt, Séries adhérentes, régularisation des suites, applications (Gauthier-Villars, Paris, 1952).

Department of Pure Mathematics,

School of General Studies,

Australian National University,

Canberra, ACT. 